\title{
Report of the American Heart Association (AHA) Scientific Sessions 2016, New Orleans
}

\author{
Makoto Amaki, MD; Nao Konagai, MD; Masashi Fujino, MD; \\ Shouji Kawakami, MD; Kazuhiro Nakao, MD; Takuya Hasegawa, MD; \\ Yasuo Sugano, MD; Yoshio Tahara, MD; Satoshi Yasuda, MD
}

\begin{abstract}
The American Heart Association (AHA) Scientific Sessions 2016 were held on November 12-16 at the Ernest N. Morial Convention Center, New Orleans, LA. This 5-day event featured cardiovascular clinical practice covering all aspects of basic, clinical, population, and translational content. One of the hot topics at AHA 2016 was precision medicine. The key presentations and highlights from the AHA Scientific Sessions 2016, including "precision medicine" as one of the hot topics, are herein reported.
\end{abstract}

Key Words: American Heart Association; Japanese Circulation Society; Late-breaking clinical trials; Scientific Sessions

$\mathbf{T}$ he American Heart Association (AHA) Scientific Sessions 2016 were held on November 12-16 at the Ernest N. Morial Convention Center, New Orleans, LA, USA. New Orleans is an attractive city that is one of the top 10 most-visited cities in the United States. The city was established by French colonists and is strongly influenced by their European culture. It is well known for its distinct French and Spanish Creole architecture, as well as its cross-cultural and multilingual heritage. New Orleans is also famous for its music, and in particular as the birthplace of jazz. On November 14, we experienced the largest full moon since 1948, the most "super" supermoon of our lifetimes. This year, the AHA conference attracted more than 18,000 participants, including physicians, research scientists, students, and paramedical personnel, from more than 100 countries (Figure 1A-E). In addition, 2 million medical professionals participated virtually in lectures and discussions about basic, translational, clinical, and population science. This 5-day event featured cardiovascular (CV) clinical practice covering all aspects of basic, clinical, population, and translational content. Here, we report the key presentations and highlights from the AHA Scientific Sessions 2016.

\section{Opening Session: Launching the AHA Precision Medicine Platform}

In the opening session (Figure 2A), the AHA President, Steven R. Houser, PhD, shared how his father's illness influenced his decision to pursue a career in CV research. In the Opening Session, the AHA and Amazon Web Services announced the creation of the AHA Precision Medicine Platform, a cloud-based marketplace for sharing the data that scientists use as fuel in the fight against CV disease, the Number 1 killer in the world. "We are changing the way research is done and believe the Precision Medicine Platform is what's needed to establish a new paradigm for scientific collaboration," said AHA CEO Nancy Brown. In addition, the AHA announced the launch of the My Research Legacy network, which is being built in conjunction with the Broad Institute of Harvard University and the Massachusetts Institute of Technology. The AHA and the Broad Institute also announced plans for the first use of the network: a pilot study of 2,000 people who survived a heart attack, stroke, atrial fibrillation (AF), aortic dissection, or systolic heart failure (HF) suffered between the ages of 21 and 50. "Throughout history, far too many lives have been impacted before the age of 50 by CV diseases and stroke," Brown said.

\section{AHA/Japanese Circulation Society (JCS) Joint Session}

The JCS maintains a close relationship with the AHA conference every year. ${ }^{1-4}$ This year, the theme of the JCS/ AHA-ReSS (Resuscitation Science Symposium) Joint Session, organized by Dr. Benjamin Abella from the University of Pennsylvania and Dr. Yoshio Tahara from the National Cerebral and Cardiovascular Center, was extracorporeal CPR (cardiopulmonary resuscitation). In this session, Dr. Tomoyuki Endo from Tohoku Medical and Pharmaceutical University gave a talk about comprehensive PCAS (post-cardiac arrest syndrome) training in Japan, including extracorporeal CPR simulation with a low-cost vascular model. Dr. David Gaieski from Thomas Jefferson University described the approach in the US to implementation and training of extracorporeal CPR (Figure 3A,B). The JCS also provided a booth in the Science

The opinions expressed in this article are not necessarily those of the editors or of the Japanese Circulation Society.

Received November 29, 2016; accepted November 30, 2016; released online December 10, 2016

Department of Cardiovascular Medicine, National Cerebral and Cardiovascular Center, Suita, Japan

Mailing address: Satoshi Yasuda, MD, Department of Cardiovascular Medicine, National Cerebral and Cardiovascular Center,

5-7-1 Fujishiro-dai, Suita 565-8565, Japan. E-mail: yasuda.satoshi.hp@ncvc.go.jp

ISSN-1346-9843 All rights are reserved to the Japanese Circulation Society. For permissions, please e-mail: cj@j-circ.or.jp 

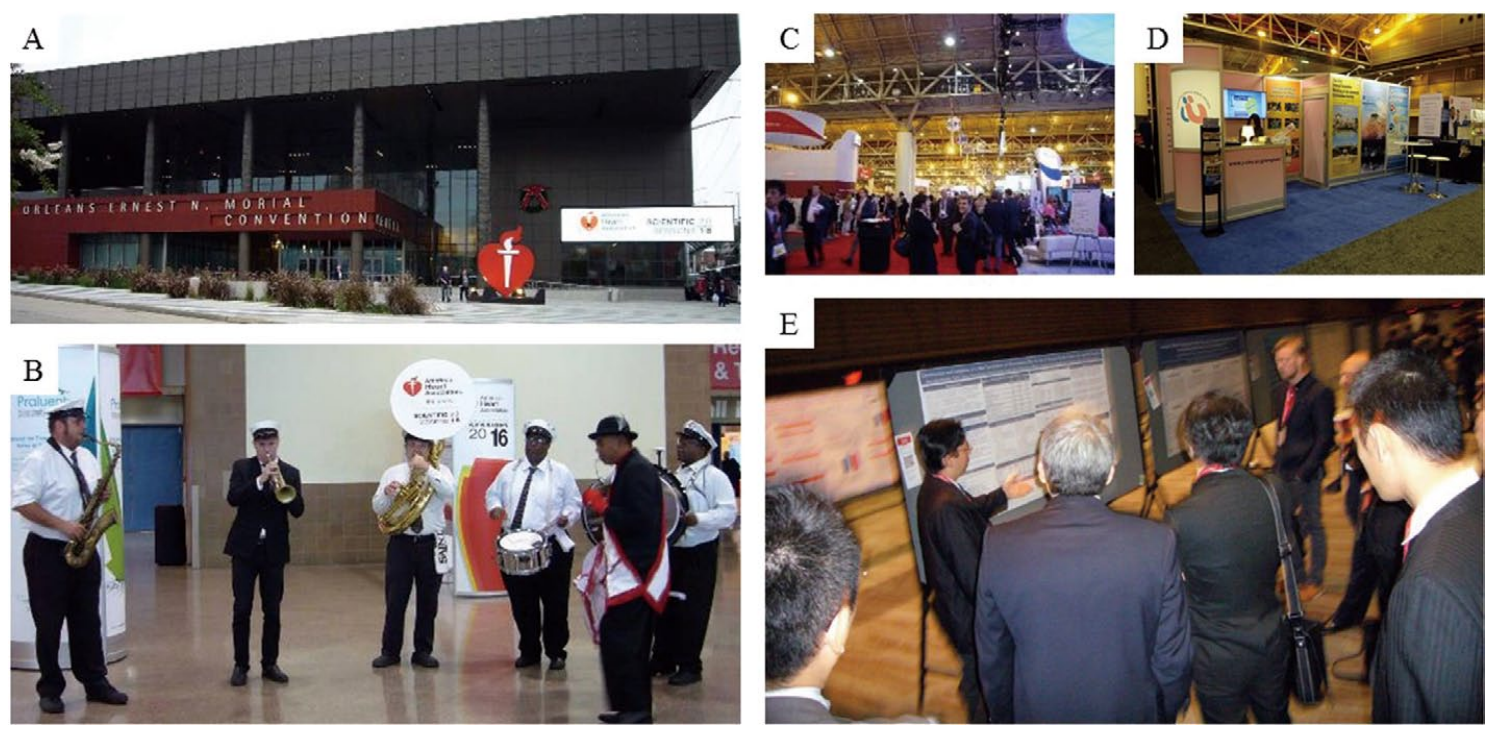

Figure 1. The 2016 American Heart Association Scientific Sessions were held at the New Orleans Ernest N. Morial Convention Center (A). New Orleans is also the birthplace of the jazz (B). The Science and Technology Hall (C). JCS booth in the Exhibition Hall (D). At the poster session (E).

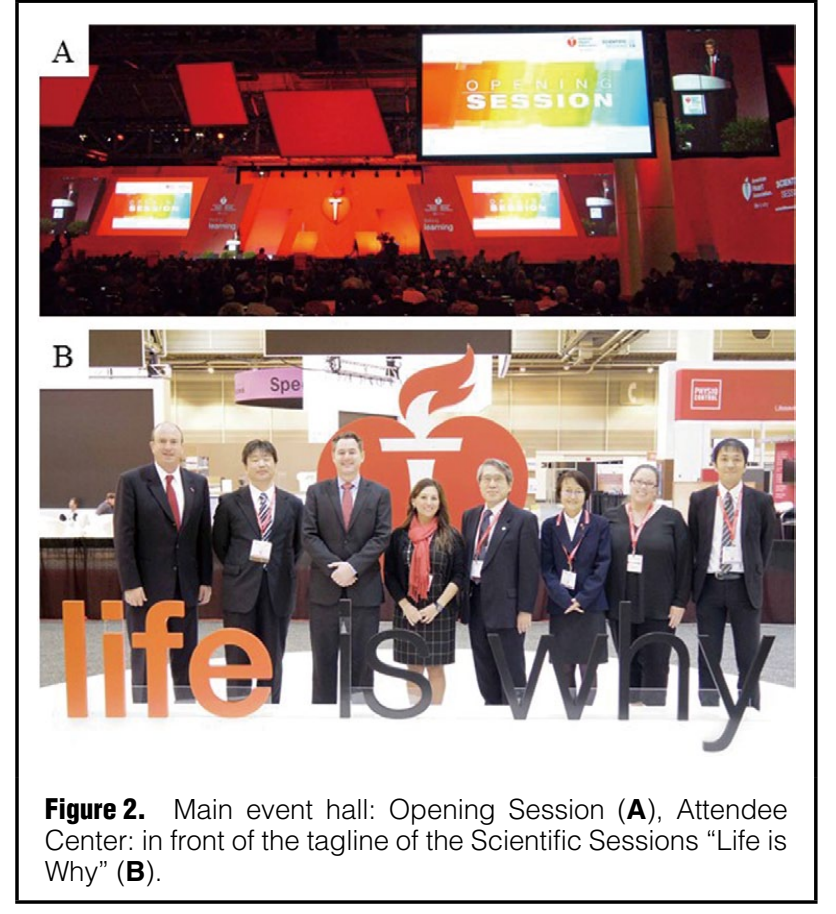

and Technology Hall, which advertised JCS activities to the AHA attendees. In the JCS booth, Dr. Endo demonstrated the Endo circuit for extracorporeal CPR training (Figure 1D). The JCS/AHA Joint Session focusing on recent advances in CV genetics was organized by Dr. Toshihiro Tanaka from Tokyo Medical and Dental University and Dr. Christopher Newton-Cheh from Massachusetts General Hospital. Dr. Kaoru Ito from Harvard Medical School described a high-throughput splicing analysis that revealed

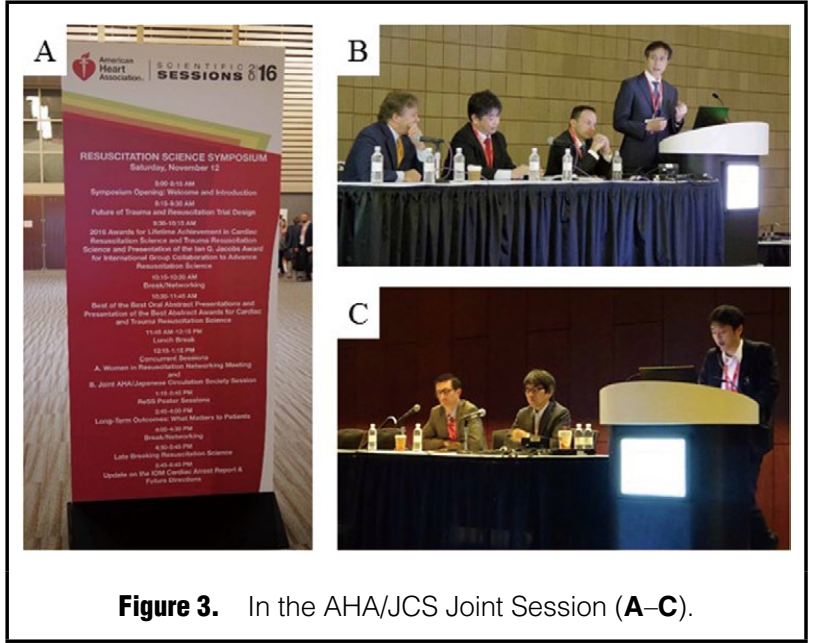

that silent mutations in $L M N A$ and $M Y B P C 3$ affect RNA splicing and cause pathogenicity. Dr. Hayato Tada from Kanazawa University described the rare and common genetic variants associated with plasma lipids and coronary artery disease. Dr. Sekar Kathiresan from Massachusetts General Hospital gave a presentation on translating results from GAWAS to the clinic. Finally, Dr. Nathan Stitziel from Washington University discussed the genetic causes of premature coronary artery disease.

\section{Late-Breaking Clinical Trials}

\section{Big Trials for Big Questions}

EUCLID: Effects of Ticagrelor Compared With Clopidogrel in Patients With Peripheral Artery Disease This randomized double-blind, double-dummy, event-driven trial was designed 
to compare ticagrelor and clopidogrel in the prevention of $\mathrm{CV}$ events in patients with symptomatic peripheral artery disease in 28 countries. ${ }^{5}$ Investigators randomized patients to ticagrelor $90 \mathrm{mg}$ twice daily or clopidogrel $75 \mathrm{mg}$ daily; eligibility was determined based on an abnormal anklebrachial index $(\mathrm{ABI}) \leq 0.80$ or prior lower extremity revascularization. Of a total of 13,885 patients with a mean age of 66 years $(72 \%$ men), 57\% were randomized based on prior revascularization and $43 \%$ based on an abnormal ABI. Of the randomized patients, $12 \%$ had a prior history of stroke or TIA, $18 \%$ prior myocardial infarction, and $23 \%$ prior coronary revascularization. The primary efficacy endpoint was the rate of major adverse cardiac events, including CV death, myocardial infarction, or ischemic stroke. This endpoint did not differ significantly between patients treated with ticagrelor and those treated with clopidogrel for 3 years (hazard ratio $1.02,95 \%$ confidence interval [CI] 0.92-1.13; $\mathrm{P}=0.65)$; in addition, there was no difference in the rates of safety outcomes, assessed by TIMI bleeding criteria. The conclusions were that ticagrelor is not superior to clopidogrel for the reduction of $\mathrm{CV}$ events, and that major bleeding occurred at similar rates in patients treated with ticagrelor or clopidogrel.

PRECISION: Cardiovascular Outcomes With Celecoxib vs. Ibuprofen or Naproxen The purpose of the Prospective Randomized Evaluation of Celecoxib Integrated Safety vs. Ibuprofen or Naproxen (PRECISION) Trial was noninferiority assessment of the $\mathrm{CV}$ risk of celecoxib vs. 2 widely used non-selective nonsteroidal antiinflammatory drugs (NSAIDs), naproxen and ibuprofen, in patients with osteoarthritis or rheumatoid arthritis. ${ }^{6}$ This trial was a double-blind, triple-dummy, randomized multicenter noninferiority study, using a 3-arm parallel group design. Study participants were 24,081 patients with osteoarthritis or rheumatoid arthritis who chronically required daily NSAIDs, at 924 sites in 13 countries. Subjects were randomized to celecoxib $209 \pm 37 \mathrm{mg}$, ibuprofen $2,045 \pm 246 \mathrm{mg}$, or naproxen $852 \pm 103 \mathrm{mg}$. Patients were provided with daily esomeprazole for gastroprotection. The primary endpoint was the composite of $\mathrm{CV}$ death, nonfatal myocardial infarction, or nonfatal stroke. In the intention-to-treat analyses, a primary outcome event occurred in 188 patients in the celecoxib group $(2.3 \%)$, in 201 patients in the naproxen group $(2.5 \%)$, and in 218 patients in the ibuprofen group $(2.7 \%)$ (hazard ratio for celecoxib vs. naproxen, 0.93 ; 95\% CI, 0.76-1.13; hazard ratio for celecoxib vs. ibuprofen, $0.85 ; 95 \%$ CI, $0.70-1.04 ; \mathrm{P}<0.001$ for noninferiority in both comparisons). The conclusion was that primary endpoint outcomes were similar for these drugs at these doses.

HOPE3: the Effect of Blood Pressure and Cholesterol Lowering on Congestion According to results from the Heart Outcomes Prevention Evaluation-3 (HOPE-3) trial, treating hypertension and elevated low-density lipoprotein cholesterol (LDL-C) late in life does not slow cognitive or functional decline. Lead author Jackie Bosch, MD, from McMaster University in Hamilton, Ontario, Canada, noted that earlier this year, the HOPE-3 researchers reported the trial's CV outcomes: reducing blood pressure and statins both significantly decreased the rate of CV events. ${ }^{7}$ The researchers used questionnaires at baseline and during follow-up to evaluate the effects of these interventions on cognitive and functional decline. Of the 12,705 participants who were randomized, 3,086 were 70 or older at baseline. Of this older cohort, 1,626 completed both the baseline and study-end questionnaire. The primary outcomes were decline in processing speed and changes in function. This study provided information on the effects of blood pressure reduction, lipid reduction, and their combination on cognitive and functional changes after 5.6 years of intervention. Cognitive decline was not prevented by long-term (5.6 years) cholesterol or blood pressure reduction in patients over 70 years of age who were at moderate risk for $\mathrm{CV}$ disease.

TRUE AHF: Short- and Long-Term Effects of Immediate Vasodilator Therapy in Acutely Decompensated Heart Failure Results from the Trial of Ularitide's Efficacy and Safety in Patients with Acute Heart Failure (TRUE-AHF) suggest that the standard model of AHF is incomplete, according to lead author Milton Packer, MD, from the Baylor Heart and Vascular Institute at Baylor University Medical Center in Dallas. The trial assessed whether, in patients with AHF, the urgent administration of the synthetic natriuretic peptide ularitide in doses sufficient to provide meaningful decongestion could decrease reduce the long-term risk of CV death. TRUE-AHF is the largest trial of AHF and involves the earliest treatment: a mean of $6.1 \mathrm{~h}$ after unplanned hospitalization or emergency department visit for acutely decompensated HF. Patients were randomized to a 48-hinfusion of ularitide or placebo, and followed for a mean of 15 months. Only 1 of the 2,157 randomized patients was lost to follow-up. The results showed that ularitide had the anticipated effect of reducing blood pressure and markers of cardiac distension during infusion, which in turn decreased the risk and number of in-hospital HF events. Although immediate vasodilator therapy in AHF reduced congestion and cardiac wall stress, the long-term risk of $\mathrm{CV}$ death was not reduced $(\mathrm{P}=0.75){ }^{8}$

\section{Pioneering the Future of HeART Interventions \\ ART: Randomized Comparison of Single vs. Bilateral Internal Mammary Artery Grafting in 2,102 Patients: Effects on Major Cardiovascular Outcomes After 5 Years of Follow-up Previous} observational studies showed that the a bilateral internal mammary artery (BIMA) approach may further improve long-term outcomes in comparison with single internal mammary artery (SIMA) plus vein grafts in patients with multivessel coronary artery disease who undergo coronary artery bypass grafting (CABG). The Arterial Revascularisation Trial (ART) was a multicenter study in which patients scheduled for CABG were randomized on clinical criteria to SIMA $(1,554)$ or BIMA $(1,448)$. The primary outcome was any-cause death. Secondary endpoints included the composite of death, myocardial infarction, stroke, and additional safety outcomes.

At 5 years of follow-up the death rate in the SIMA group was $8.4 \%$ vs. $8.7 \%$ in BIMA (HR 1.04 ; $95 \%$ CI $0.82-1.33$; $\mathrm{P}=\mathrm{NS})$ and the composite of death, MI, and stroke was $12.7 \%$ in SIMA vs. $12.2 \%$ in BIMA (HR 0.97; $95 \%$ CI 0.79-1.18; $\mathrm{P}=\mathrm{NS})$. Adjustments for covariates yielded the same results. By contrast, sternal wound infection and reconstruction were more frequent in the BIMA group (1.9\% vs. $3.5 \%[\mathrm{P}=0.005]: 0.6 \%$.vs $1.9 \%[\mathrm{P}=0.002]$, respectively). At 5 years of follow-up, BIMA provided similar clinical outcomes to SIMA with some early excess of sternal wound complications. Follow-up to 10 years is ongoing to determine whether BIMA provides longer term benefits, because vein graft failure becomes more common after 5 years. 
FUTURE: The Functional Testing Underlying Coronary Revascularization (FUTURE) Study - A "Real-World" Comparison of Fractional Flow Reserve-Guided Management vs. Conventional Management in Multivessel Coronary Artery Disease Patients Although previous studies suggested that fractional flow-reserve (FFR)-guided revascularization by percutaneous coronary intervention (PCI) can improve clinical outcomes, there is no hard evidence assessing the use of FFR in a "real-world" setting for therapeutic management of patients with multivessel coronary artery disease. This study was performed to investigate whether FFR-guided therapeutic management including medical treatment only, PCI, or CABG surgery is superior to conventional management in patients with multivessel disease. This investigation was a multicenter open, randomized clinical trial. Patients with multivessel disease including the left anterior descending (LAD) coronary artery were randomized to either FFR-guided management or traditional angio-guided management. The primary endpoint was a composite of major adverse $\mathrm{CV}$ events, including all-cause death, non-fatal myocardial infarction, stroke, and repeat coronary revascularization at 1 year. Although the trial was scheduled to include 1,728 patients, the independent data safety monitoring board recommended that study enrollment be stopped because of the significantly greater mortality in the FFR-group (7 vs. 17: HR 2.39; $\mathrm{P}=0.019$ ). As of the day of presentation, follow-up had indicated a persistent but not significant $(\mathrm{P}=0.11)$ difference in 12-month all-cause mortality (797 patients). The 1-year follow up for all patients is currently ongoing.

PIONEER AF-PCI: an Open-Label, Randomized, Controlled, Multicenter Study Exploring Two TreatmeNt Strategies of Rivaroxaban and a Dose-Adjusted Oral Vitamin K Antagonist Treatment Strategy in Subjects With Atrial Fibrillation Who Undergo Percutaneous Coronary Intervention Patients with AF who undergo PCI are often treated with triple therapy using an oral anticoagulant plus dual antiplatelet therapy, but both the optimal combination and duration of therapy remains uncertain. Previous randomized studies suggested that a dual-pathway therapeutic approach using a factor Xa inhibitor and a single antiplatelet agent may be superior. To test this hypothesis, 2,124 AF patients undergoing PCI were randomized in a 1:1:1 ratio to receive either rivaroxaban $15 \mathrm{mg}$ q.d. plus thienopyridine for 12 months, rivaroxaban $2.5 \mathrm{mg}$ b.i.d., or dose-adjusted vitamin $\mathrm{K}$ antagonist. The investigators found consistent bleeding reductions in patients receiving either dose of rivaroxaban. The number need to treat patients to avoid a significant bleed using either of these strategies was 10 or 11 patients, and 10-15 patients to prevent a hospitalization.

GARY: Patients at Intermediate Surgical Risk Undergoing Isolated Interventional or Surgical Aortic Valve Replacement for Severe Symptomatic Aortic Valve Stenosis - 1-Year Results From the German Aortic Valve Registry Transcatheter aortic valve implantation (TAVI) is the recommended treatment for high-risk patients who are inoperable or at high surgical risk. PARTNER II showed that TAVI was not inferior to surgical aortic valve replacement (SAVR) in intermediate risk patients (STS score 4-8\%). However, randomized control trials usually cover selected populations and may not reflect real-world populations. Researchers screened 49,660 patients enrolled in the German Aortic Valve Registry (GARY), covering $87 \%$ of all aortic valve procedures performed in Germany between January 2011 and December 2013, and identified 5,997 patients at inter- mediate risk who underwent isolated TAVI or SAVR $(4,101$ TAVI; 1,596 SAVR). TAVI patients were older (81.8 vs. 75.9 years, $\mathrm{P}<0.01)$, predominately female $(61.6 \%$ vs. $54.1 \%$, $\mathrm{P}<0.01$ ), had higher symptom scores (NYHA $\geq 3,83.7 \%$ vs. $72.4 \%, \mathrm{P}<0.01)$, and more severe medical conditions. The strongest predictors for TAVI were low calcium score, prior cardiac decompensation, coronary artery disease, pulmonary hypertension, lack of prior $\mathrm{CABG}$, and tricuspid valve regurgitation. All-cause mortality at 1 year was much higher in patients who underwent TAVI than in those who underwent SAVR (16.6 vs. 8.9, $\mathrm{P}<0.01)$. Even with a propensity score analysis, there were significant differences in all-cause mortality, in favor of surgery (transfemoral and transapical, 4.6\%; transfemoral only, 3.4\%).

\section{Insights From New Therapeutic Trials for Lipids \\ ORION 1: Inhibition of PCSK9 Synthesis via RNA Interference} - 90-Day Data From Orion-1, a Multi-Center Phase-2 Randomized Controlled Trial Current therapies targeting PCSK9 use monoclonal antibodies, which require injections every $2-4$ weeks. Inclisiran, a novel RNA interference synthetic double-stranded oligonucleotide (PSCK9si), is less expensive, with potential for sustained suppression of PCSK9 production with 2-3 injections yearly. The ORION-1 trial is a phase 2 double-blind randomized controlled trial comparing placebo with 6 different dosing regimens of PCSKsi. A total of 501 patients with atherosclerotic CV disease (ASCVD) or high ASCVD under maximally tolerated statin therapy were enrolled into 2 study arms. The single-dose arm compared 200, 300, and $500 \mathrm{mg}$ doses of inclisiran with placebo, and the 2-dose arm compared two 100,200 , and $300 \mathrm{mg}$ doses of inclisiran with placebo. Inclisiran achieves a $50 \%$ reduction in LDL-C with 1 dose for up to 90 days, gradually rising to around $45 \%$ reduction at 150 days; 2 doses achieve $55-60 \%$ reduction in LDL-C for up to 180 days. Treatment emergency adverse events were similar across all groups. This study demonstrates the potential clinical ability of PCSK9si, administered quarterly or bi-annually, to provide sustained reduction in LDL-C, supporting initiation of a phase III trial.

GLAGOV: Effect of Evolocumab on Progression of Coronary Atherosclerosis in Statin-Treated Patients - A PlaceboControlled Intravascular Ultrasound Trial PCSK9 inhibitors markedly and incrementally reduce the level of LDL-C when used in combination with statins. This study sought to determine whether treatment with PCSK9 inhibitor modifies coronary atherosclerotic disease progression. ${ }^{9}$ In this phase III double-blind placebo-controlled trial, 968 statin-treated patients with angiographic coronary diseases were randomized to receive PCSK9 inhibitor evolocumab or placebo monthly for 76 weeks, in addition to statins. Coronary plaque volume was measured by serial IVUS imaging. Mean LDL-C levels were lower in the evolocumab group than in the placebo group $(93.0$ vs. $36.6 \mathrm{mg} / \mathrm{dL}$, $\mathrm{P}<0.001)$ with no safety issues identified. Atheroma volume declined $5.8 \mathrm{~mm}^{3}(\approx 1 \%)$ with evolocumab, a greater decrease than that observed with placebo $(\mathrm{P}<0.001)$. In the placebo arm, $47.3 \%$ of patients exhibited regression in atheroma volume, vs. $64.3 \%$ of patients in the evolocumab arm $(\mathrm{P}<0.001)$. Addition of the PCSK inhibitor evolocumab to statin therapy yielded a greater reduction in LDL level and atheroma regression. The GLAGOV is the first study to use IVUS to demonstrate regression in coronary plaque volume. 
AEGIS I: The Safety and Tolerability of CSL112, a Reconstituted, Infusible, Human ApoA-I, After Acute Myocardial Infarction - The ApoA-I Event Reduction in Ischemic Syndromes I (AEGISI) Trial CSL112 is an infusible formulation of human apoA-I that has been shown to increase high-density lipoprotein-mediated cholesterol efflux capacity and regress atherosclerotic disease. Apo-I Event Reduction in Ischemic Syndromes-I (AEGIS-I), a multicenter placebo-controlled, dose-ranging randomized phase $2 \mathrm{~b}$ trial, evaluated the safety profile of CSL112 and characterized its pharmacokinetic (PD)/pharmacodynamics (PK) properties in acute myocardial infarction (AMI) patients. A total of 1,258 patients were randomized to receive either of 2 doses of CSL112 (2g or $6 \mathrm{~g}$ ) or placebo, weekly for 4 consecutive weeks. Co-primary safety endpoints were occurrence of either a hepatic safety event or a renal safety event. The 4-weekly infusions of CSL112 at both low and high dose within 7 days after AMI were feasible, were not associated with alterations in either liver or kidney function or other significant safety concerns, and were associated with immediate improvements in cholesterol efflux capacity.

Ionis-Angptl3-IRx, an Antisense Inhibitor to AngiopoietinLike Protein 3 [angptl3] Reduces Plasma Angptl3 and Lipids in Healthy Volunteers With Elevated Triglycerides Complete loss of function of angiopoietin-like 3 (ANGPTL3) results in familial combined hypolipidemia, manifesting as a reduction of all lipoproteins except for lipoprotein(a). IONIS-ANGPTL3-LRx, an antisense oligonucleotide (ASO) against ANGPTL3 conjugated to the $\mathrm{N}$-acetyl galactosamine complex, specifically targets the ASO to the asialoglycoprotein receptor on hepatocytes. This randomized, placebo-controlled phase 1 study was conducted to examine the effect of IONIS-ANGPTL3-LRx in healthy volunteers with elevated TG, as well as to evaluate its safety, tolerability, PK, and PD. A total of 44 patients were given a single ascending dose from 20 to $80 \mathrm{mg}$ or weekly multiple ascending doses from 10 to $60 \mathrm{mg}$, subcutaneously for 6 weeks. IONISANGPLT3-LRx reduced plasma levels of ANGPTL3 by $83 \%$, and significant mean reductions were noted in TGs $(-66 \%)$, apoC-III $(-68 \%)$, LDL-C $(-35 \%)$, TC $(-36 \%)$, and non-HDL-C $(-40 \%)$. There were no safety concerns related to target reduction or drug administration.

MILANO-PILOT: Impact of Infusion of an ApoA-IMilano HDL Mimetic on Regression of Coronary Atherosclerosis in Acute Coronary Syndrome Patients Infusions of an HDL mimetic containing the naturally occurring variant apoA-IMilano promoted plaque regression in patients with acute coronary syndromes (ACS). This phase 2, double-blind, placebocontrolled trial was conducted to evaluate the effects of infusing HDL mimetic containing ApoA-IMilano (MDCO216) on regression of coronary atherosclerosis in patients with recent ACS. A total of 120 patients with ACS events within the previous 14 days were randomized to receive either five $20 \mathrm{mg} / \mathrm{kg}$ weekly infusions of MDCO-216 or placebo. The primary endpoint was the percent change in atheroma volume (PAV), determined by IVUS, in a single coronary artery with a maximum stenosis of $20-50 \%$ from baseline to the end of the study. There was no difference in PAV change between the 2 groups (median $-0.8 \%$ [interquartile range $-2.3,0.2]$ in placebo group vs. $-0.5 \%[-1.8$, $0.5]$ in MDCO-216 group; $\mathrm{P}=0.10)$. In addition, there were no differences in total atheroma volume or percent of patients exhibiting regression in PAV. MDCO-216 did not significantly change the PAV for ACS patients in this pilot study.
Guiding the Momentum to Effect HF Outcomes: Ironing Out the Wrinkles

REDUCE LAP-HF Study: Transcatheter Interatrial Shunt Device Provides Sustained Clinical Benefit at 1 Year in Patients With Preserved or Mildly Reduced Ejection Fraction The REDUCE LAP-HF study is a multicenter, prospective, nonrandomized, open-label single-arm study designed to investigate the safety and clinical and hemodynamic benefits of an interatrial septal shunt device that reduces left atrial pressure in patients with HF with preserved ejection fraction. ${ }^{10}$ A total of 64 patients with left ventricular ejection fraction $\geq 40 \%$, New York Heart Association (NYHA) class II-IV, elevated pulmonary capillary wedge pressure ( $\geq 15 \mathrm{mmHg}$ at rest or $\geq 25 \mathrm{mmHg}$ during supine bicycle exercise) participated. At 1 year after interatrial septal shunt device implantation, there were sustained improvements in NYHA class, quality of life (Minnesota Living with Heart Failure score), and 6-minute walk distance. Invasive hemodynamic studies performed in a subset of patients demonstrated a sustained reduction in the workload-corrected exercise pulmonary capillary wedge pressure. Survival at 1 year was $95 \%$, and there was no evidence of device-related complications. These results provide evidence of safety and sustained clinical benefit in HF patients with preserved ejection fraction 1 year after interatrial septal shunt device implantation. Randomized, blinded studies are underway to confirm these observations.

ATHENA HF: Aldosterone Targeted Neurohormonal Combined with Natriuresis Therapy in Heart Failure Trial The aim of this study was to test the hypothesis that use of high-dose spironolactone in patients with acute HF (AHF) leads to greater reductions in NT-proBNP levels from randomization to $96 \mathrm{~h}$. In this randomized, double blind, placebocontrolled multicenter trial, a total of 360 patients were enrolled. Patients were randomized to receive either usual care or usual care plus $100 \mathrm{mg}$ spironolactone; 182 received high-dose spironolactone, and 178 received usual care (132 placebo, 46 continued low-dose spironolactone). Mean age was 65 years; $74 \%$ of patients had HF with reduced ejection fraction, and estimated glomerular filtration rate was $61.5 \mathrm{~mL} / \mathrm{min} / 1.73 \mathrm{~m}^{2}$. No significant difference in worsening renal function or hyperkalemia was observed between the 2 groups, suggesting tolerance of high-dose spironolactone, but $100 \mathrm{mg} /$ day spironolactone for $96 \mathrm{~h}$ in AHF patients did not achieve its primary aim of reducing the NT-proBNP level to a greater extent than usual care. Additionally, none of the secondary endpoints (dyspnea relief, clinical congestion, net urine output, weight loss, or clinical events) differed between the 2 groups. The data do not support the routine use of high-dose spironolactone in AHF.

IRONOUT HF: Oral Iron Repletion Effects on Oxygen Up Take in Heart Failure Despite growing recognition of the functional and prognostic significance of iron deficiency in $\mathrm{HF}$, the utility of oral iron supplementation is unknown. The aim of this study was to test the hypothesis that oral iron polysaccharide is superior to oral placebo in improving exercise capacity (peak $\dot{\mathrm{VO}}_{2}$ ) in patients with $\mathrm{HF}$ and reduced ejection fraction (HFrEF) and iron deficiency at 16 weeks.

In this multicenter, randomized, double-blinded placebocontrolled trial of oral iron polysaccharide $(300 \mathrm{mg} /$ day $)$ compared with matching placebo, 225 patients (mean age 63 years, female $36 \%$, NYHA II/III $66 \% / 33 \%$ ) with HFrEF (LVEF $<40 \%$ ) and iron deficiency, defined as a serum ferritin 15 and $100 \mathrm{ng} / \mathrm{mL}$ or serum ferritin $100-299 \mathrm{ng} / \mathrm{mL}$ 
with transferrin saturation (Tsat) $<20 \%$ were enrolled and randomized: 111 to oral iron, and 114 to placebo. The primary endpoint, change in peak $\mathrm{V}_{2}$, did not differ between groups. In ancillary analyses, oral iron only modestly improved iron measurements. In conclusion, high-dose oral iron minimally filled iron stores and did not improve exercise capacity in HFrEF patients, raising questions about the sufficiency of oral iron therapies in patients with HF.

EFFECT HF: Effect of Ferric Carboxymaltose on Exercise Capacity in Patients With Iron Deficiency and Chronic Heart Failure (EFFECT HF) - A Randomized, Controlled Study This prospective, randomized, controlled, open-label, international multicenter 2-arm trial was designed to study the effects of treating iron deficiency in chronic HF patients with intravenous ferric carboxymaltose (FCM). A total of 172 stable HF (NYHA II-III) patients with reduced exercise capacity were randomized to receive either intravenous FCM or standard care for 24 weeks. The primary endpoint was peak $\dot{\mathrm{VO}}_{2}$ at 24 weeks. Irrespective of baseline anemia, a significantly beneficial effect on peak $\dot{\mathrm{V}}_{2}$ was observed in the intravenous FCM arm relative to the standard care arm. These findings confirm and extend the findings of previous studies showing that treatment with FCM improves exercise capacity and symptoms in patients with HF and iron deficiency.

MOMENTUM 3: Multicenter Study of MagLev Technology in Patients Undergoing Mechanical Circulatory Support Therapy With HeartMate 3 - Primary Results of the Short-Term (6-Month) Cohort This prospective, randomized, controlled trial was designed to compare the HeartMate3 to HeartMate2 for noninferiority in patients with advanced HF and who were candidates for a left-assisted ventricular device (LVAD). The primary endpoint was a composite including survival to transplant, recovery, or LVAD support free of debilitating stroke or reoperation of the pump at 6 months. At 6 months, the composite endpoint was significantly improved for HeartMate3 relative to HeartMate2 (HeartMate3 vs. HeartMate2: $86.2 \%$ vs. $76.8 \%$; $\mathrm{P}<0.001$ ).

MultiSENSE: a Multi-Sensor Algorithm Predicts Heart Failure Events in Patients With Implanted Devices In this study, 1-year follow-up data combining heart sounds, respiration, thoracic impedance, heart rate and activity were evaluated to determine whether an algorithm using data from sensors from implanted devices can detect changes in HF status in patients with $\mathrm{HF}$ and reduced ejection fraction. The $2 \mathrm{co}-$ primary endpoints, sensitivity performance goal (PG) of $>40 \%$ unexplained alert rate (UAR) PG of $<2$ alerts per patient-year, were tested with a 2 -sided $95 \% \mathrm{CI}$ and evaluated using 320 patient-years of follow-up data and 50 adjudicated usable $\mathrm{HF}$ events, including $\mathrm{HF}$ admissions or unscheduled visits with augmented intravenous HF treatment, in the Test Set cohort. With an observed sensitivity of $70 \%$ (lower two-sided 95\% CI: $55.4 \%$ ) and UAR of 1.47 (upper 2-sided 95\% CI: 1.65), both endpoints were met. The multi-sensor HF diagnostic algorithm significantly exceeded its prespecified endpoints, demonstrating compelling performance for worsening HF detection.

\section{Closing Remarks}

This event featured 5 days of the best in science and CV clinical practice covering all aspects of basic, clinical, population, and translational content. The scope and quality of the scientific exchange make the Scientific Sessions the premier $\mathrm{CV}$ research and instructional meeting in the world. One of the hot topics at AHA 2016 was precision medicine, defined as "an emerging approach for disease treatment and prevention that takes into account individual variability in genes, environment and lifestyle for each person." Precision medicine and big-data analysis could enable cardiologists to make decisions based on highly individualized variables, not just on generalized trial results or guidelines. We hope that our report will inspire young Japanese cardiologists to attend future AHA Scientific Sessions.

\section{Acknowledgments}

We thank all the doctors who participated in the AHA/JCS Joint Session and generously permitted us to use images from their presentations. We also thank Shizuka Yamada and Takashi Amano from the JCS for sharing images.

\section{Disclosures}

None.

\section{References}

1. Fujita J. Report of the American Heart Association (AHA) Scientific Sessions 2012, Los Angeles. Circ J 2013; 77: 35-40.

2. Ishimori N, Kinugawa S, Yamada S, Yokoshiki H, Mitsuyama H, Tsutsui H. Report of the American Heart Association (AHA) Scientific Sessions 2013, Dallas. Circ J 2014; 78: 51-56.

3. Kohno T. Report of the American Heart Association (AHA) Scientific Sessions 2014, Chicago. Circ J 2015; 79: 34-40.

4. Aizawa Y, Kimura M, Kohno T, Fujita J, Fukuda K. Report of the American Heart Association (AHA) Scientific Sessions 2015, Orlando. Circ J 2016; 80: 51-57.

5. Jones WS, Baumgartner I, Hiatt WR, Heizer G, Conte MS, White CJ, et al. Ticagrelor compared with clopidogrel in patients with prior lower extremity revascularization for peripheral artery disease. Circulation, doi:10.1161/CIRCULATIONAHA.116.025880.

6. Becker MC, Wang TH, Wisniewski L, Wolski K, Libby P, Luscher TF, et al. Rationale, design, and governance of Prospective Randomized Evaluation of Celecoxib Integrated Safety versus Ibuprofen Or Naproxen (PRECISION), a cardiovascular endpoint trial of nonsteroidal antiinflammatory agents in patients with arthritis. Am Heart J 2009; 157: 606-612.

7. Yusuf S, Lonn E, Pais P, Bosch J, Lopez-Jaramillo P, Zhu J, et al. Blood-pressure and cholesterol lowering in persons without cardiovascular disease. N Engl J Med 2016; 374: 2032-2043.

8. Packer M, Holcomb R, Abraham WT, Anker S, Dickstein K, Filippatos G, et al. Rationale for and design of the TRUE-AHF trial: The effects of ularitide on the short-term clinical course and long-term mortality of patients with acute heart failure. Eur $J$ Heart Fail, doi:10.1002/ejhf.698.

9. Nicholls SJ, Puri R, Anderson T, Ballantyne CM, Cho L, Kastelein JJ, et al. Effect of evolocumab on progression of coronary disease in statin-treated patients: The GLAGOV randomized clinical trial. JAMA, doi:10.1001/jama.2016.16951.

10. Kaye DM, Hasenfuss G, Neuzil P, Post MC, Doughty R, Trochu $\mathrm{JN}$, et al. One-year outcomes after transcatheter insertion of an interatrial shunt device for the management of heart failure with preserved ejection fraction. Circ Heart Fail, doi:10.1161/ CIRCHEARTFAILURE.116.003662. 